\title{
A Comprehensive Analysis of Plasmonics-Based GaAs MSM-Photodetector for High Bandwidth-Product Responsivity
}

\author{
Narottam Das, ${ }^{1}$ Farzaneh Fadakar Masouleh, ${ }^{2}$ and Hamid Reza Mashayekhi ${ }^{2}$ \\ ${ }^{1}$ Department of Electrical and Computer Engineering, Curtin University, Perth, WA 6845, Australia \\ ${ }^{2}$ Department of Physics, University of Guilan, Rasht 41335-1914, Iran \\ Correspondence should be addressed to Narottam Das; narottam.das@curtin.edu.au
}

Received 29 April 2013; Revised 10 August 2013; Accepted 22 August 2013

Academic Editor: Jung Huang

Copyright (c) 2013 Narottam Das et al. This is an open access article distributed under the Creative Commons Attribution License, which permits unrestricted use, distribution, and reproduction in any medium, provided the original work is properly cited.

\begin{abstract}
A detailed numerical study of subwavelength nanogratings behavior to enhance the light absorption characteristics in plasmonicbased metal-semiconductor-metal photodetectors (MSM-PDs) is performed by implementation of 2D finite-difference timedomain (FDTD) algorithm. Due to the structure design and changes in the device physical parameters, various devices with different geometries are simulated and compared. Parameters like nano-grating height and duty cycle (DC) are optimized for rectangular and taper subwavelength metal nanogratings on GaAs substrate and their impact on light absorption below the diffraction limits are confirmed. The calculated light enhancement is $\sim 32.7$-times for an optimized device in comparison with a conventional MSM-PD. This enhancement is attributed to the plasmonic effects in the near-field region.
\end{abstract}

\section{Introduction}

Periodic nanostructures can produce an efficient light transmission and absorption by excitation of surface plasmons (SPs) [1] and their potential practice (or application) in optical communication system has made them an interesting type of devices [2]. The continuing progress in plasmonic interaction with nanostructures and their outstanding design instruction in metal-semiconductor-metal photodetectors (MSM-PDs) has developed a unique context for futuregeneration optoelectronic systems, such as, optical fiber communication systems, high speed chip-to-chip interconnects, and high-speed sampling [3]. The conventional MSM-PD is a symmetrical device which is equivalent to two back-to-back connected Schottky diodes on a semiconductor substrate [4], such as GaAs which as a direct band gap semiconductor collects and emits photons more efficiently than indirect semiconductors such as Si and Ge [5]. To create a Schottky junction, some essential properties must be satisfied, such as type and quality of metal and semiconductor along with the shape and size of the interface [6]. A significant amount of illumination on top of a conventional MSM-PD is reflected, and the light absorption is reduced considerably in device's active region. Fabrication of nanogratings on top of the metal fingers on conventional MSM-PDs can avoid the unwanted reflection and exhibit notable light transmission. By tailoring the electrodes structure surface with metal nanogratings, MSM-PDs can be modified for the light absorption, and the modification process strongly depends on the corrugation parameters. Recently, different shapes of one-dimensional (1D) nanostructured surfaces have been developed in which noble metals, such as gold $(\mathrm{Au})$, are used for nanograting structuring. When an electric field (or a voltage) is applied between the electrodes and the device's active region is under illumination, then the electric carriers (i.e., electrons and holes) are generated and drifted towards the opposite electrodes due to the electric field, and can form a photocurrent. Improvement of recombination between electrons and holes can lead to enhance the light absorption in the subwavelength aperture region $[3,7,8]$. Over the decade, an interesting effect of light interacting with metallic structures has been revealed. For sufficiently small nanograting period, higher order diffractions are suppressed and only the zero order diffraction is present. There has been a considerable and growing interest to clarify all phenomena corresponding to the improvement in light absorption via nanoscale structuring recently [9-12]. The main motivation is to come up with a practical method for avoiding the undesirable light reflection from the surface of the electrodes and enhance the light transmission 


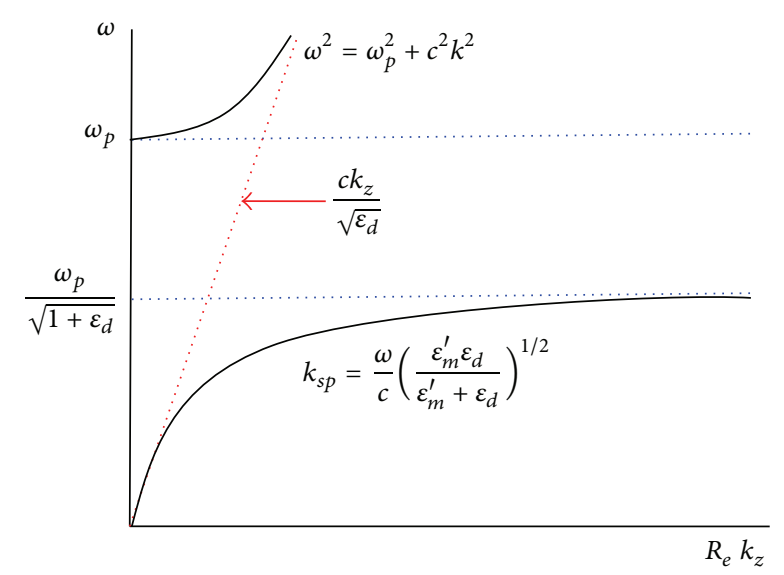

FIGURE 1: The dispersion relation of nonradiative SPs on the right of the light line and dispersion relation of radiative SPs on the left of the light line.

efficiency through the subwavelength aperture. It is useful to describe the SP dispersion relation as shown in Figure 1. To have propagating bound SPs, the wave vector component must be real along the interface. Any light cannot be used for SP generation, because the real part of the wave vector in the $z$-direction exceeds that of free light $(\omega=c k)$ as shown in Figure 1, and a coupling mechanism is required.

In the presence of a transverse magnetic (TM) polarized light, the SPs exist along a metal-dielectric interface. There are several techniques to excite the SP waves, such as prism coupling and grating coupling. For the case of semiconductors, prism coupling is not very much advantageous. The attention should be paid to prism refractive index which is hardly higher than popular semiconductors used for corresponding researches $[13,14]$. In particular, this study presents the effects of nanograting structures on the MSM-PD device. Optimization of subwavelength nanogratings shape and pitch is very much important to generate the zero-order diffraction waves. Thus, the subwavelength nanogratings can be represented as a homogeneous medium with optical properties determined by the nanograting geometry [15]. When the nanograting period is within the order of the light wavelength, the light wave may be resonant and reflects into the structure; hence, the higher diffraction orders will be suppressed, and resonant reflection occurs for the zero-order diffraction waves [16-18]. The metallic nanogratings can exhibit absorption anomalies. One of these particularly remarkable anomalies is observed for $p$-polarized light only and is due to the surface plasmon polariton (SPP) excitations [19]. The light incident on the onedimensional metallic nanogratings composed of subwavelength slits is converted into the propagating SPPs that can absorb the light efficiently in extremely thin (10 nm to more than $100 \mathrm{~nm}$ 's thick) layers. The coupling of light to the structure in the form of the SPPs is obtained with the utility of periodicity. There are two distinct mechanisms to produce the transmission of light in 1D metal nanograting with narrow slits, which are the excitation of horizontal and vertical surface resonances. The horizontal surface resonances are excited by the periodic structure of the nanogratings. The vertical surface resonances have relations with Fabry-Perot resonances of the fundamental TM guided wave in the slits [1]. Two vertical resonances existing on the slit walls can compose a fundamental waveguide mode which satisfies the Fabry-Perot condition and reflects repeatedly from both ends of the slit. Therefore, the guided modes can be excited along vertical direction, evanescent coupling mechanism accounts for this activation [20]. The responsibility of these two effects on extraordinary optical transmission (EOT) is still under debate. However, it is indicated that both mechanisms are important in the EOT [21]. Concentrating the light in small areas (plasmonic lenses) with the assistance of extremely thin layers [22], the EOT is a unique phenomenon which is induced by the SP assisted multiple diffractions, coupling the SPs into the aperture, and conversion back into near-field at the exit side of the aperture. The role of SP modes is essential to clarify the EOT of light through the slits. In the EOT, the aperture transmits more light than the standard aperture theory. The EOT can occur whenever some specific conditions are satisfied, that is, the slit width must be much smaller than the incident light wavelength, the periodicity has to be in the range of wavelength and it can lead to outstanding results if the light is normally incident to the structure's surface. Subwavelength apertures have also been used to concentrate light efficiently into the deep subwavelength regions.

A considerable development has been reported for the EOT through metallic grating with very narrow slits [23]. The SPPs and subwavelength apertures in metallic thin films are always involved with this phenomenon but the details are still under investigation $[7,9,16]$. The SPs can be efficiently excited in the nanostructured noble metals since they almost have free-electron behavior [24]. The noble metal nanotextured structures have special properties to produce localized regions of high energy concentration and show larger enhancement for extraordinary optical absorption (EOA). This effect and its underlying mechanism have important applications in photolithography and near-field microscopy.

The absorption enhancement caused by the excitation of SPPs is associated with the incident photons and their interaction with the nanogratings, whereas Fabry-Perot resonances are included in transmission absorption process through the subwavelength slits. In addition, the light is mostly reemitted from a very small area surrounding the aperture which is associated with the properties of the Fabry-Perot cavity resonances for symmetric SPP modes of the slit.

The main objective of this research is to find out the radiation behavior through the nanoscale device. Finite-difference time-domain (FDTD) simulation tool is a full wave modeling technique that demonstrates significant light-capturing performance through the periodic $1 \mathrm{D}$ slit arrays [25] for the design of ultrafast MSM-PDs [3]. The FDTD algorithm was originally proposed by Yee in 1966 [26] and introduces a modeling technique to solve the Maxwell's curl equations in time domain. It can directly calculate the value of $E$ (electric field intensity) and $H$ (magnetic field intensity) at different mesh points in the computational domain denoted by $(i, j, k)$ with respect to Ampere and Faraday's laws (Maxwell equations). Since then, it has been used for several applications 
and many extensions of the basic algorithm developed [27]. Currently, the FDTD method is widely used for electromagnetism computations, such as fields and resonant modes.

\section{Nanostructured MSM Photodetector Design}

A plasmonics-based MSM-PD structure has three separate regions or parts, namely, the incident region (the metal nanogratings), the grating area and the subwavelength apertures region, and the transmission region (the substrate). The nanoscale gaps between the interdigitated electrodes in the MSM-PDs result in a huge increase in bandwidth and reduction in dark current, whereas the conventional PIN photodetectors with similar sized active areas are unable to achieve that amount of light.

The momentum of SPs can be easily changed by adding thin layers of material on the metal surface or by changing the dielectric constant of the material deposited on it. Here, the gold $\mathrm{(Au}$ ) metal nanogratings were deposited on top of the underlayer containing subwavelength apertures, and the underlayer is just on the semiconductor (GaAs) substrates while the whole structure is surrounded by air. When the surface is corrugated, the electromagnetic radiation can be coupled with the SPPs. Incident photons on a corrugated surface can induce diffraction by addition or subtraction of multiples of nanograting vector, $k_{g}=2 \pi / \Lambda$, where $\Lambda$ is the grating period. Therefore, the nanocorrugations should satisfy the dispersion relation by providing the momentum difference as in (1), which is governed by the momentum selection rules (i.e., occurring only at a specific angle for a given wavelength). For the TM polarization, diffraction orders will be in the classical mount where all diffraction orders are in the plane of incident. The SPP charge density waves are confined to the metal-dielectric interface and can only be excited with $p$ polarized electromagnetic radiation, where the magnetic field is along the $1 \mathrm{D}$ nanograting corrugations $\left(H_{y}\right)$. This means, in case of a one-dimensional nanograting, the corrugations are the grooves of infinite length in the $y$-direction. Here, the nanogratings are called the continuous nanogratings with rectangular (or tapered) cross-section because they are placed on a thin layer of the same metal to fabricate the modified device structure. The linearly polarized incident radiation of light with appropriate frequency and angle of incident satisfies the dispersion relation equation; hence, the SPP diffraction occurs [28]. The coupling of incident electromagnetic wave to the SPs on the nanograting assisted MSM-PD structure surface is shown in Figure 2.

The TM polarized wave is required to excite the propagating SP waves, when the $z$-component of the light $k$-vector matches with the SP $k$-vector, because only the TM polarization has electric field component in the same direction as surface normal. To represent the influence of electric field in organizing electric charges and dipoles in the medium, we introduce electric displacement field, $D=\varepsilon_{0} \varepsilon_{r} E$, between two isotropic media where $\varepsilon_{0}$ is the vacuum permittivity and $\varepsilon_{r}$ is the relative (dielectric) permittivity. Here, the gold has complex dielectric permittivity in the form of $\varepsilon_{m}=\varepsilon_{m}^{\prime}+i \varepsilon_{m}^{\prime \prime}$, from which the real part has been used. The dielectric permittivity of air is $\varepsilon_{d}$. The real part of gold dielectric

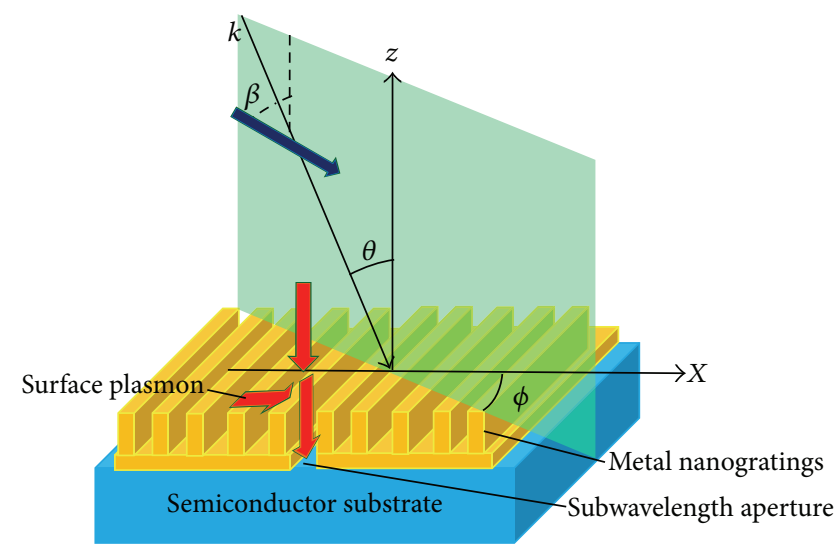

FIgURE 2: A symmetric diagram of a plasmonic-based MSM-PD structure with rectangular-shaped nanogratings on interdigitated electrodes and semiconductor substrate. Here, the gold nanogratings are on the substrate made from GaAs. EOT will occur as a result of SP-light interactions.

permittivity has negative value at optical wavelength. The electric displacement field derived from Maxwell's equation is continuous across the interface. With the continuous normal component of $D$ across the interface and the permittivity sign difference for metal and dielectric, the electric field changes direction passing through two different media. This characteristic will only be satisfied if there is a normal component for electric field across two regions. The polarization is described with regard to the electric field configuration of the incident light with respect to the surface normal (angle $\beta$ ) as shown in Figure 2. The wave is purely TE polarized, when $\beta=\pi / 2$, and purely TM polarized, when $\beta=0$. The angle $\phi$ represents the direction of the plane of incidence, such that the nanograting is in the classical mount in this model, where all the diffracted orders remain in the $x-z$ plane, and the plane of incidence is normal to the nanograting grooves $(\phi=0)$. Besides, when the grooves are parallel to the plane of incidence, the nanograting is in the conical mount $(\phi=90)$.

In periodic subwavelength structures, the nanogratings are deposited on top of the underlayer from the same metal (such as gold). The absorption enhancement can be achieved by the SPP resonant excitations in the subwavelength region. Here, the FDTD method is used to specify the TM polarized plane wave via Poynting vector. The SPPs are evanescent waves, which are generated by the interaction between the surface electron densities and the electromagnetic fields whilst trapping the light power inside the surface. Since the SP modes have longer wave vectors than the light waves with the same energy, the SP waves are nonradiative on smooth metallic surfaces and cannot propagate in nonmetallic media. One way to excite the SPPs is the nanograting coupling technique in which the incident radiation is coupled into the SPPs using a periodic surface corrugation [17]. The nanograting grooves are perpendicular to the $x$-direction and its dimensions and geometry are optimized to couple the light near the design wavelength, that is, providing the missing momentum in order to make the SPPs propagate along the $z$-direction. In a metal-dielectric interface, the SPP wave vector matching 
condition for a metal nanograting can be defined with some changes to the well-known prism resonance condition [29]. Hence, the SPP propagating constant or wave vector with metal nanograting period of $\Lambda$ is given by $[13,16,30]$

$$
\frac{\omega}{c} \sin (\theta) \pm \frac{2 \pi l}{\Lambda}=k_{\mathrm{sp}}=\frac{\omega}{c} \sqrt{\frac{\varepsilon_{m}^{\prime} \varepsilon_{d}}{\varepsilon_{m}^{\prime}+\varepsilon_{d}}},
$$

where $\Lambda$ is the metal nanograting period, $\omega$ is the angular frequency of the incident light wave, $c$ is the speed of light in vacuum, $\theta$ is the angle between the incident light and the normal, and $l$ is an integer.

This relation illustrates that the wave vector of a given frequency is smaller than the SPP wave vector; therefore, the light wave vector should increase with the support of a coupling mechanism to provide SPPs which is satisfied by nanograting structure. To trigger SPPs, the dielectric permittivity has to change sign in the metal-dielectric interface. The metal permittivity has to be complex, $\varepsilon_{m}=\varepsilon_{m}^{\prime}+i \varepsilon_{m}^{\prime \prime}$. Therefore, the wave vector is complex. The SPP damping while propagating along the interface will be determined by the imaginary part of the wave vector parallel component. The researchers demonstrated their results for noble metals, such as gold and silver in metal-dielectric interface [31]. The values of dielectric constant vary for different frequency ranges. We are interested in metals with the large negative real part and a very small imaginary contribution to the dielectric constant for the design wavelength, such as gold. Therefore, there will be a high field confinement at the interface while the losses remain minimum. When the plasmonic excitations occur, the left side of (1) matches the wave vector of the excited SPP $\left(k_{\mathrm{spp}}\right)$, that is, the equivalence of the interaction of incident radiation and $l$ th diffracted order with the wave vector of SP at the interface.

\section{Noble Metals and Lorentz-Drude Model}

Nowadays, it is clear that the real metals play a very important role in EOT, because their effect is essential for the SP excitation. The real metal is characterized by the frequency dependent dielectric permittivity, $\varepsilon_{m}=\varepsilon_{m}^{\prime}+i \varepsilon_{m}^{\prime \prime}$. It consists of a negative real part $\varepsilon_{m}^{\prime}$ and a positive imaginary part $\varepsilon_{m}^{\prime \prime}$ responsible for light absorption, which is obtained from Lorentz-Drude model. The letter $i$ is an imaginary unity, that is, $\sqrt{-1}$ and $\varepsilon_{d}$ is the dielectric permittivity of the incidence medium, here it is air. The condition for the existence of the SP on a flat air-metal interface, $\varepsilon_{m}^{\prime}<-1, \varepsilon_{m}^{\prime \prime} \ll\left|\varepsilon_{m}^{\prime}\right|$, is fulfilled for many metals, including the silver and gold $[32,33]$.

Dielectric and magnetic properties of the material with nanotextured structure can easily be determined by the implementation of Lorentz-Drude model [34]. Below plasma frequency, the good conductors like silver $(\mathrm{Ag})$ and gold $(\mathrm{Au})$ have negative values for the real part of complex dielectric constant. Therefore, it is necessary to define an appropriate model to specify the dielectric properties of the materials. A complex dielectric function for some metals and surface plasmas which have good agreement with the experimentally measured results can be expressed in the following form [35]:

$$
\varepsilon_{r}(\omega)=\varepsilon_{r}^{f}(\omega)+\varepsilon_{r}^{b}(\omega),
$$

where the term $\varepsilon_{r}(\omega)$ is the complex dielectric function for metals, $\varepsilon_{r}^{f}(\omega)$ is referred to as free electron effects, and $\varepsilon_{r}^{b}(\omega)$ is associated with bound electron effects. This model takes both the intraband, $\varepsilon_{r}^{f}(\omega)$, and interband, $\varepsilon_{r}^{b}(\omega)$, effects into account for simulations. The former, Drude model, can describe the transport properties of electrons in good conductors and the later, Lorentz model, is a semiquantum model describing bound electron effects. The Drude and Lorentz model in frequency domain can be written as in the following form of (3) and (4), respectively.

$$
\begin{gathered}
\varepsilon_{r}^{f}(\omega)=1+\frac{\Omega_{p}^{2}}{i \omega \Gamma_{0}-\omega^{2}}, \\
\varepsilon_{r}^{b}(\omega)=\sum_{m=1}^{M} \frac{G_{m} \omega_{p}^{2}}{\omega_{m}^{2}-\omega^{2}+i \omega \Gamma_{m}},
\end{gathered}
$$

where $\Omega_{p}=G_{0}{ }^{1 / 2} \omega_{p}$ is the plasma frequency associated with intraband transitions with oscillator strength $G_{0}$ and damping constant $\Gamma_{0}$, while $m$ is the number of oscillators with frequency $\omega_{m}$, and $1 / \Gamma_{m}$ is the oscillator lifetime [36]. The following equation accounts for the complex index of refraction and dielectric constant of materials and can be represented as a combined form [37]:

$$
\varepsilon_{r}(\omega)=\varepsilon_{r, \infty}+\sum_{m=0}^{M} \frac{G_{m} \Omega_{m}^{2}}{\omega_{m}^{2}-\omega^{2}+i \omega \Gamma_{m}},
$$

where $\varepsilon_{r, \infty}$ is the relative permittivity at infinite frequency, $G_{m}$ is the strength of each resonance term, $\Omega_{m}$ is the plasma frequency, $\omega_{m}$ is the resonant frequency, and $\Gamma_{m}$ is the damping factor or the collision frequency.

OptiFDTD is the first software to employ the LorentzDrude model into the FDTD algorithm. Here, the LorentzDrude model for gold is solved with 6 resonant frequencies (multipole dispersion). The simulation is performed over a constant plasma frequency which depends on the density of charge carriers with the amount of $0.137188 E+17$. The resonant frequency changes according to the resonance strength. The Lorentz-Drude material parameters are listed in Table 1.

\section{Results and Discussion}

To study the mentioned effects on the plasmonic-based MSM-PD structure, we adopted the FDTD algorithm with the aid of Opti-FDTD software package developed by Optiwave Inc. Nanograting assisted MSM-PD is introduced with an important feature of the EOT. The concept of plasmonic lens is represented which utilizes plasmonic effects to produce SPPs and funnel the energy toward the central focus point. Hence, the device is enabled to enhance the collection of photocurrent into the near field region [38,39]. A set of numerical simulations are performed to calculate the Poynting vector which is particularly useful in quality light absorption. Only on metal surfaces, electromagnetic wave can propagate in the form of SPPs. We used nanograting coupling technique to enhance the device performance. The numerical analysis of the corrugated metal surfaces with different nanograting 
TABLE 1: Lorentz-Drude parameters for gold (measured in radians per second).

\begin{tabular}{lccc}
\hline Term & Strength & Resonant frequency & Damping frequency \\
\hline 0 & 0.7600 & $0.000000 E+00$ & $0.805202 E+14$ \\
1 & 0.0240 & $0.630488 E+15$ & $0.366139 E+15$ \\
2 & 0.0100 & $0.126098 E+16$ & $0.524141 E+15$ \\
3 & 0.0710 & $0.451065 E+16$ & $0.132175 E+16$ \\
4 & 0.6010 & $0.653885 E+16$ & $0.378901 E+16$ \\
5 & 4.3840 & $0.202364 E+17$ & $0.336362 E+16$ \\
\hline
\end{tabular}

shapes is implemented and their performances are compared. Presuming that the full power flow through the slits reaches to the GaAs substrate, the optimal absorbed radiation ratio to the whole incident power can be perceived by a dimensionless quantity, namely, the light absorption enhancement factor (LAEF) defined as the ratio of normalized light transmission through the device with the nanogratings to that of the conventional type device without the nanogratings [40]. The term LAEF shows the impact of nanogratings on the quality of light flux transmitted into the active region of the device comparing to the conventional device without the nanogratings.

We calculated the amount of light flux transmitted into the slit for the plasmonic-based MSM-PD structure sets with the rectangular, trapezoidal, and triangular nanograting profiles and their behavior are compared. Focused ion beam (FIB) lithography technique is used to develop nanofabrications and nanogratings are successfully patterned in plasmonic-based MSM-PDs. The observation of SEM images represents the nanogratings with taper walls rather than the rectangular nanogratings $[3,41]$ and the simulation results confirm the capability of some trapezoidal nanogratings under special design which enhances the LAEF more than the rectangular one, while the reverse is true for some other structures. Here, several numerical simulation results for the enhancement of optical absorption in MSM-PDs are presented.

4.1. Effect of Nanograting Height on the LAEF. In this subsection, we have calculated the amount of light flux transmitted into the slit for 4 different rectangular nanograting heights and presented the LAEF spectra in Figure 3. The peak wavelength behaves like a sinusoidal manner, and wavelength $(\lambda)$ is red shifted as the ridge's height increases. The duty cycle is $60 \%$ while the nanograting period is kept constant at $810 \mathrm{~nm}$. The subwavelength aperture width and height are kept constant at $50 \mathrm{~nm}$, and $20 \mathrm{~nm}$, respectively. The TM mode of light was perpendicularly incident on the groove profiles. The height of the ridge is an effective parameter as different sets of results show significant changes in the amount of light transmitted into the active area of the MSM-PD with the variation of nanogratings height. There are some interpretations to analyze the curves. The SPPs coupling process and the following expected absorption can easily occur for higher gratings and reduces after certain heights. This light absorption has a maximum for a specific wavelength in the spectra which varies in different heights. For a mesh step size of $\delta x=10 \mathrm{~nm}$, the green curve represents about 26 -fold absorption enhancement, the maximum LAEF for $120 \mathrm{~nm}$ nanograting height, Figure 3. For the wavelengths higher than the peak, the amount of LAEF decreases and the reason might be the incident light coupling into radiative SPs, more than bound ones.

Several sets of numerical analysis are carried out to illustrate the effect of trapezoidal-shaped nanograting height and demonstrated the height at which the resonance transmission is maximum. The simulations are performed for 4 different nanograting heights of $100,120,140$, and $160 \mathrm{~nm}$ with the subwavelength aperture width of $50 \mathrm{~nm}$ and the underlayer thickness of $20 \mathrm{~nm}$. The results are shown in Figure 4. The LAEF is 26.3 times in its maximum for the green curve representing the $140 \mathrm{~nm}$ nanograting height for the duty cycle of $60 \%$. Hence, tapered nanogratings height at maximum light absorption is higher than the optimum height of its rectangular counterpart while its resonant wavelength is blue shifted.

4.2. Effect of Duty Cycles on the LAEF. This subsection discussed the effect of duty cycles on the LAEF of the nanostructured MSM-PDs. The nanograting profile which is made of gold on the GaAs substrate can change the absorption enhancement. Hence, for 2 similar nanogratings with the discrepancy in profile shapes, the LAEF can be different. We specify the optimum duty cycle for trapezoidal and rectangular-shaped nanograting profiles with an optimum height of $140 \mathrm{~nm}$ and $120 \mathrm{~nm}$, respectively. The transmission spectra are calculated with $\delta x=10 \mathrm{~nm}$ mesh step in the FDTD simulation.

We calculated the LAEF spectra in rectangular-shaped nanograting structures for different duty cycles, such as from $30 \%$ to $90 \%$, as shown in Figure 5, while the subwavelength aperture width has been kept constant at $50 \mathrm{~nm}$, the underlayer thickness and the nanograting height were $20 \mathrm{~nm}$ and $120 \mathrm{~nm}$, respectively. It can be inferred that the peak wavelength is different for each specific duty cycle and the maximum LAEF occurs for $40 \%$ duty cycle with the amount of $\sim 32.7$. It is clear that the duty cycle can affect the peak wavelength as well as the amount of light flux transmitted into the active area of the MSM-PDs.

The optimum duty cycle has also been specified for trapezoidal-shaped nanograting profile with the subwavelength aperture width and thickness of $50 \mathrm{~nm}$ and $20 \mathrm{~nm}$, respectively. In this case, the maximum LAEF is obtained $~ 31.5$ for $40 \%$ DC which is the same duty cycle as the optimum DC for rectangular-shaped nanogratings.

The results shown in Figures 5 and 6 illustrate that the amount of LAEF is a function of duty cycle which grows gradually towards the $40 \%$ DC and drops down dramatically towards $90 \%$ DC for both the structures and the peak wavelengths are red-shifted for the higher duty cycles. In the case of the rectangular and trapezoidal nanograting profiles designed with their optimum nanograting height and duty cycle, nanostructures with normal walls perform better. Therefore, the amount of power (energy) transmitted into the active region not only depends on the nanogratings height 


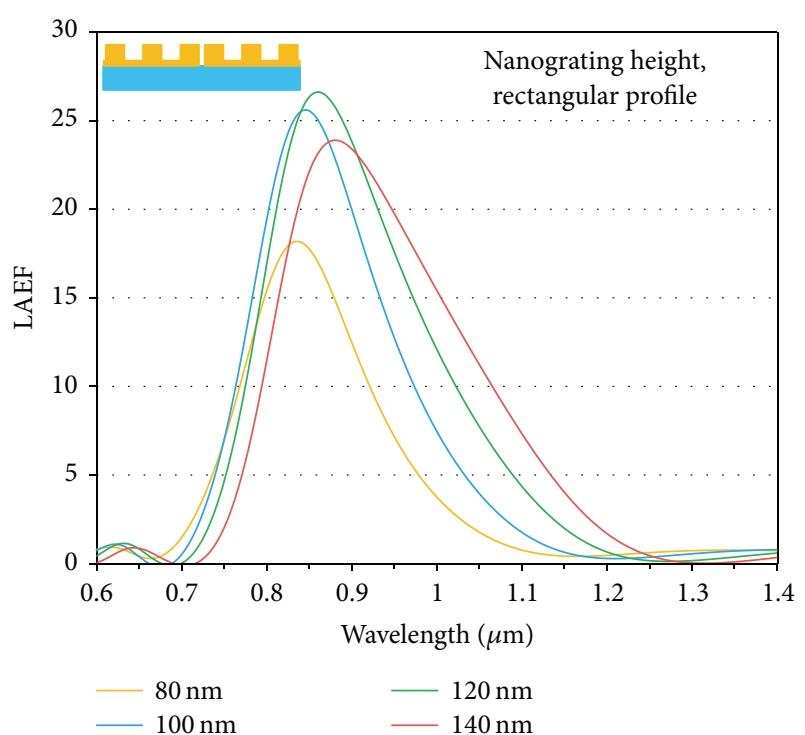

FIGURE 3: LAEF spectra for rectangular nanogratings with different heights. Here, DC is $60 \%$ and subwavelength aperture width is $50 \mathrm{~nm}$.

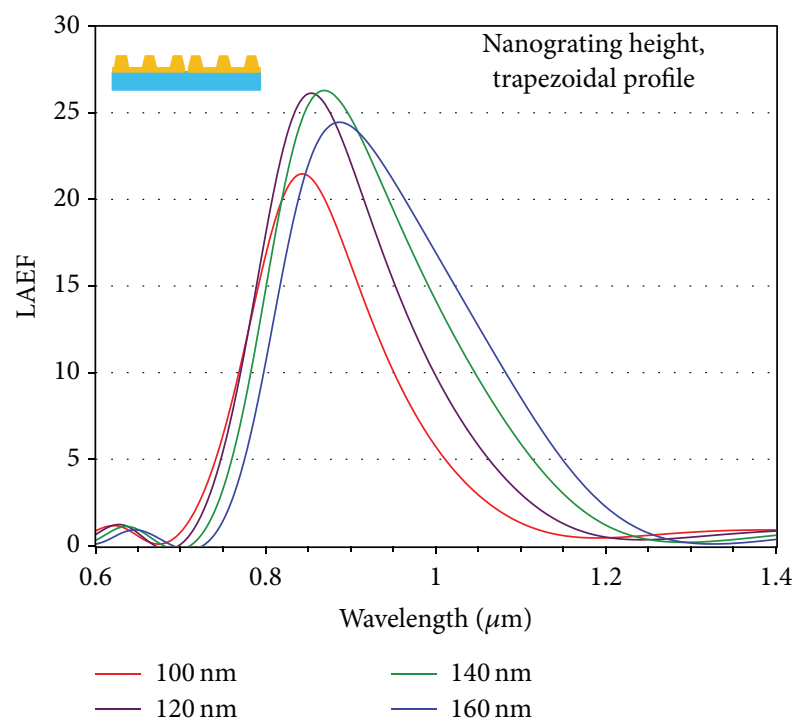

FIGURE 4: LAEF spectra for different nanograting heights of trapezoidal profiles. Here, DC is $60 \%$ and subwavelength aperture width is $50 \mathrm{~nm}$.

but also depends on the amount of duty cycles. Besides, for rectangular-shaped nanograting profile, 100\% DC indicates that the whole structure is like a conventional MSM-PD with the underlayer thickness of about $140 \mathrm{~nm}$, the resulting thickness is the sum of underlayer thickness and the nanograting height, while for trapezoidal shaped nanogratings, there will be triangular grooves between the trapezoidal ridges for $100 \%$ DC, Figure 7.

In plasmonic-based MSM photodetector design with nanogratings, more photons have the chance of penetration and concentration into the device's active region via narrow slits of nanograting assisted MSM-PDs. Therefore, it is useful

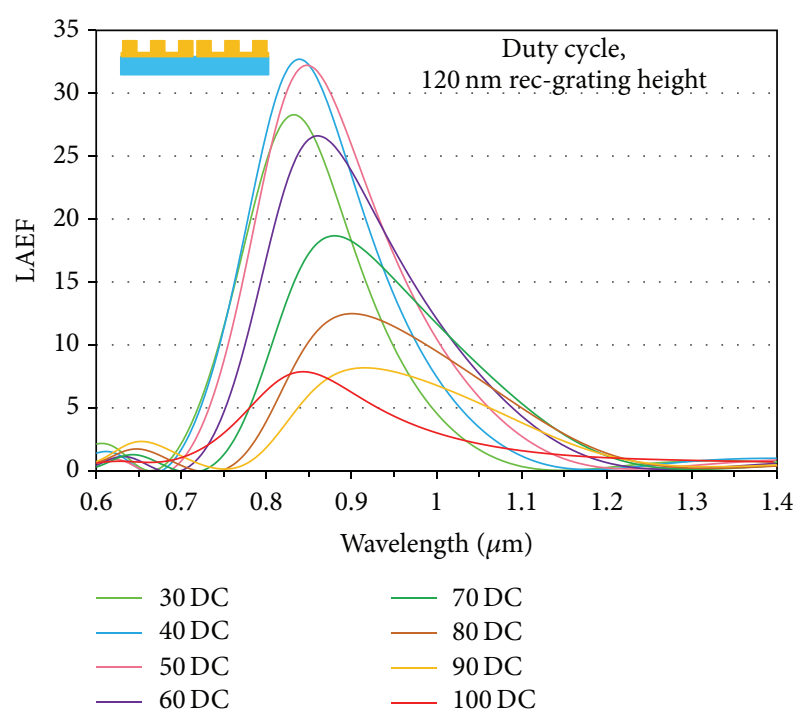

FIGURE 5: LAEF spectra for different duty cycles in MSM-PD with rectangular nanogratings. Here, the nanograting height is $120 \mathrm{~nm}$ and subwavelength aperture width is $50 \mathrm{~nm}$.

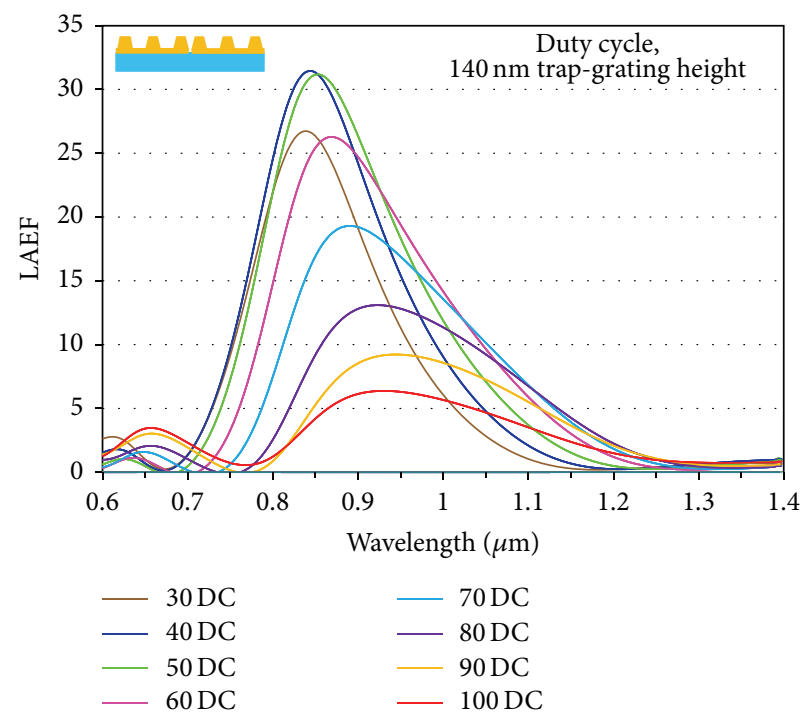

FIGURE 6: LAEF spectra for different duty cycles in trapezoidal nanograting assisted MSM-PD. Here, the nanograting height is $140 \mathrm{~nm}$ and subwavelength aperture width is $50 \mathrm{~nm}$.

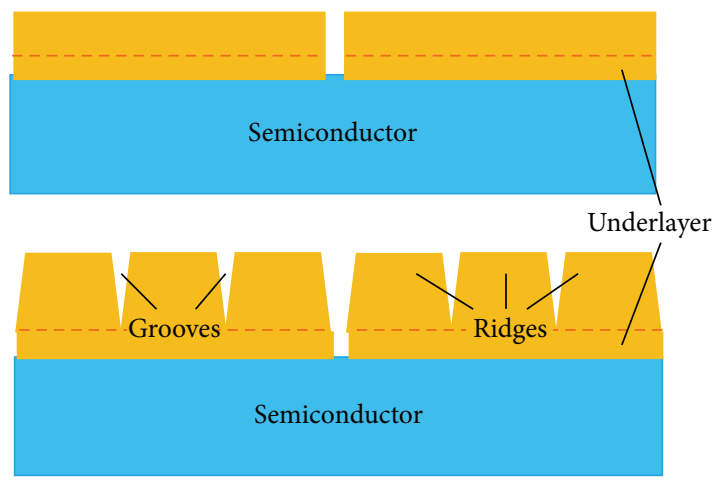

FIGURE 7: Cross-section of rectangular and trapezoidal nanogratings with duty cycle of $100 \%$. 


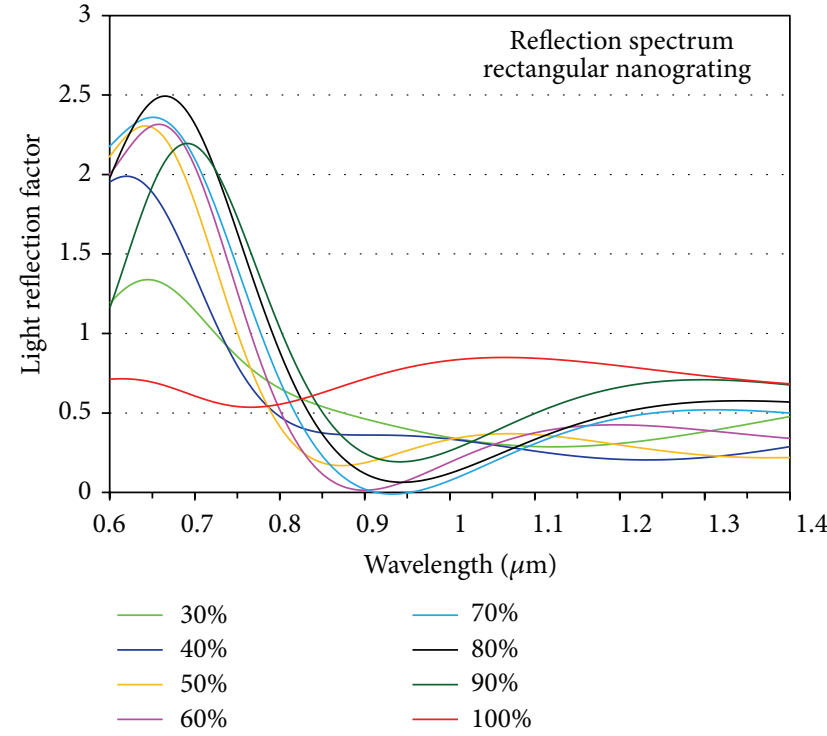

(a)

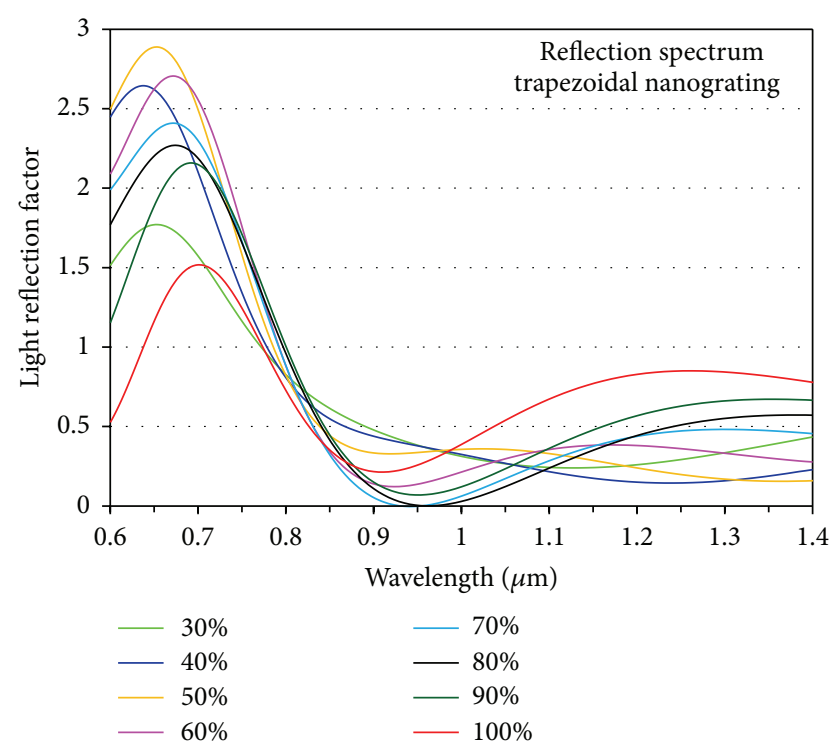

(b)

FIGURE 8: Light reflection spectra for rectangular (a) and trapezoidal (b) plasmon-assisted MSM-PDs with different duty cycles. Here, the subwavelength aperture width and heights are kept constant at $50 \mathrm{~nm}$ and $20 \mathrm{~nm}$, respectively.

to make a comparison between the amount of electromagnetic radiation which is reflected back and the amount of absorbed light in the substrate. Here, we introduce the light reflection factor which is defined as the amount of light reflected from the device with the nanogratings normalized to the amount of reflected light from the surface of conventional type device without the nanogratings. Figure 8 shows the calculated reflection spectrum of rectangular and trapezoidal nanograting profiles for normal incidence in comparison to the transmission spectrum presented for the similar devices with aforementioned parameters in Figures 5 and 6 , respectively. The curves show the reflection spectrum calculated above the central slit for different duty cycles. Less reflection is observed for nanogratings with vertical walls considering the range of the light reflection factor for both rectangular and trapezoidal nanogratings. Besides, the interval between the maximum and minimum reflection factors is $\sim 0.4$ and 1.1 for the rectangular and trapezoidal nanograting profiles, respectively. The reflectance peak grows with the increase of duty cycle up to $80 \%$ and it falls for $90 \%$ in the rectangular nanograting profile while in the case of trapezoidal nanogratings, the maximum reflectance peak is for $50 \%$ duty cycle.

4.3. Effect of Subwavelength Aperture Width on the LAEF. In this subsection, the effect of subwavelength aperture widths on the light absorption enhancement of different nanograting profiles in MSM-PDs is discussed. The aperture width is very small compared to the incident light wavelength $\left(\lambda_{0}\right)$; hence symmetric and fundamental SP modes will propagate into the slit. The simulation results for 3 different nanograting groove shapes with the variation of subwavelength aperture width, and the cross-section profiles of the grating structures are shown in Figure 9. Apart from the shape of nanogratings and

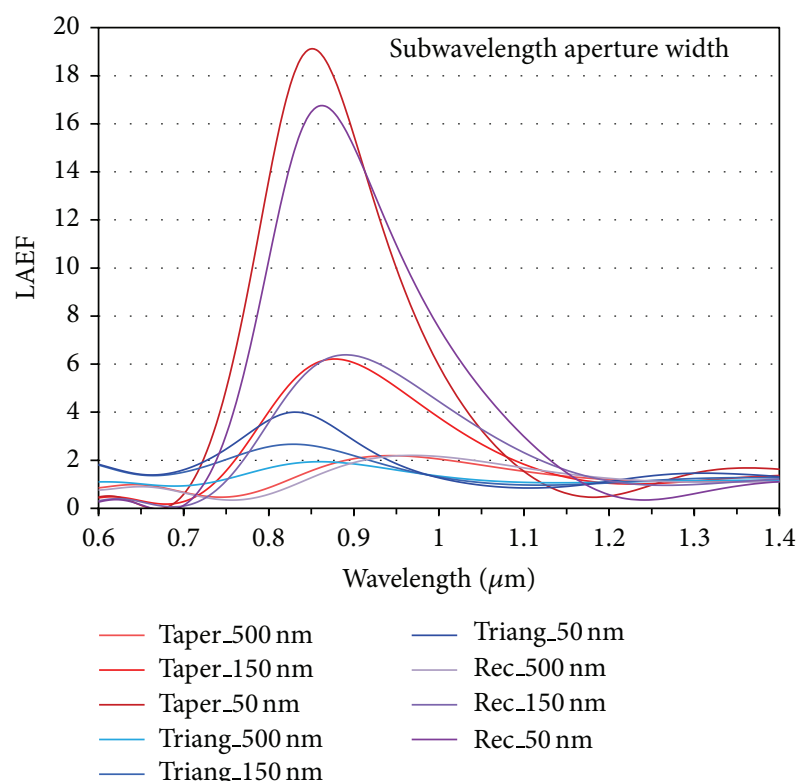

(a)

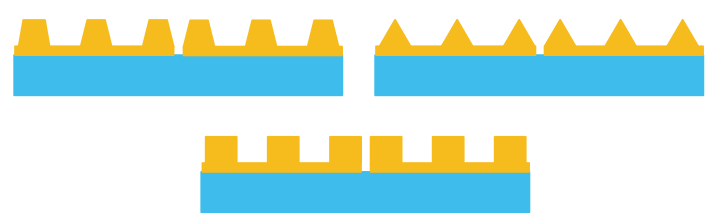

(b)

FIGURE 9: LAEF spectra for plasmon-assisted MSM-PDs with different aperture widths for rectangular, trapezoidal, and triangularshaped nanogratings. Here, the subwavelength aperture and nanograting heights are kept constant at $60 \mathrm{~nm}$ and $100 \mathrm{~nm}$, respectively. 


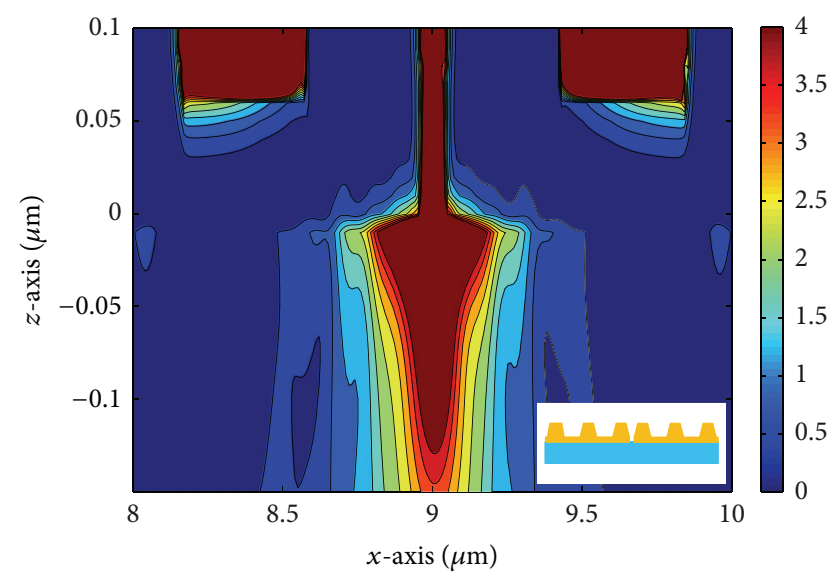

(a)

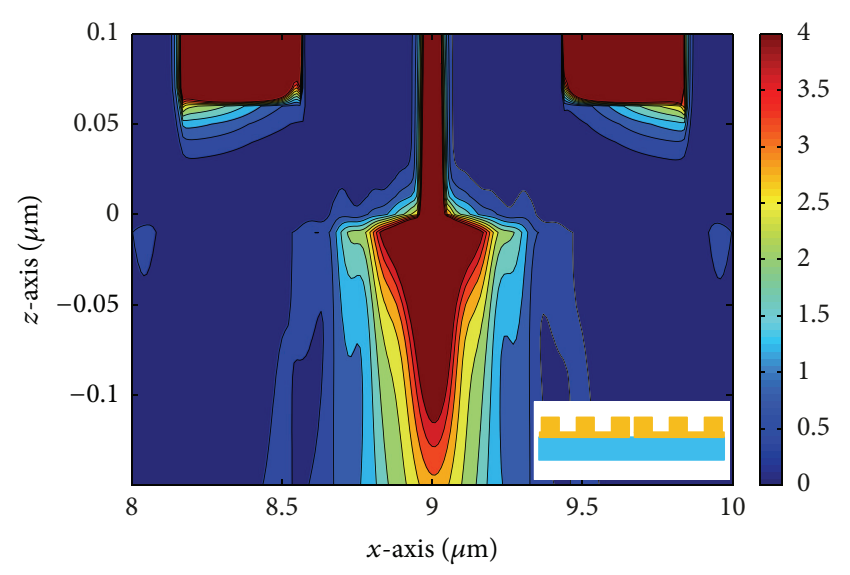

(b)

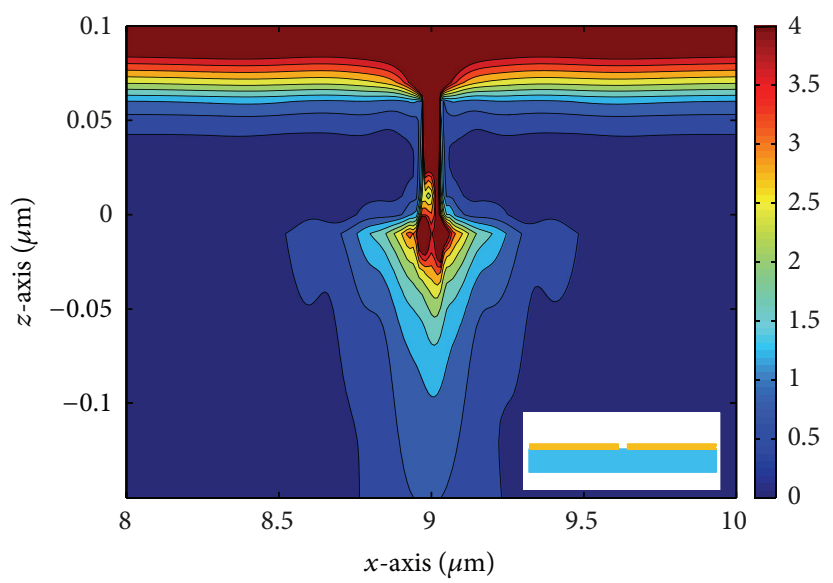

(c)

FIGURE 10: Field distribution at the cross-section of trapezoidal (a) and rectangular (b) nanograting assisted MSM-PDs besides conventional type device without nanogratings (c) at the subwavelength aperture. The calculated total electric field intensity distribution inside the GaAs substrate is shown using the following parameters: gold underlayer thickness of $60 \mathrm{~nm}$, grating period of $810 \mathrm{~nm}$, and the subwavelength aperture width of $50 \mathrm{~nm}$.

subwavelength aperture widths, other structural parameters are invariant. The nanogratings are designed with the constant period of $810 \mathrm{~nm}$ for 9 nanogratings considered on each side of the slit (subwavelength aperture). The accepted opinion is that the light transmission enhancement and improved absorption in the semiconductor substrate can be obtained when the subwavelength aperture width is much smaller than the incident light wavelength $\left(\lambda_{0}\right)$. This condition assists the metal nanogratings in the light transmission and absorption effectively. The FDTD simulation results are shown for $\delta x=10 \mathrm{~nm}$ mesh size. The LAEF spectra are presented in Figure 9 for the rectangular, trapezoidal-shaped with 0.9 aspect ratio, and triangular-shaped nanogratings. For $60 \mathrm{~nm}$ underlayer thickness, $100 \mathrm{~nm}$ nanograting height, and 50\% duty cycle the light absorption is minimum for triangularshaped nanograting. The LAEF in $150 \mathrm{~nm}$ and $500 \mathrm{~nm}$ aperture widths for the rectangular profiles is slightly higher than the trapezoidal ones, while the trapezoidal nanogratings have the ability to improve the light absorption more than the rectangular nanogratings for $50 \mathrm{~nm}$ subwavelength aperture, the narrowest slit width. It has been stated that the light is gradually compacted from the top to the bottom of the tapered grooves; therefore, the field is gradually enhanced more effectively than the grooves with normal walls. We have plotted the electric field distribution of these 2 structures to confirm how this variation influences the amount of LAEF from 19 to 16.7 .

Figure 10 demonstrates the increase of light absorption in trapezoidal nanograting grooves compared to the rectangular nanogratings with the slit width of $50 \mathrm{~nm}$. Also the light absorption is calculated within the cross-section of a basic MSM-PD without nanogratings on the electrodes to prove its incompetence in the absence of plasmon polariton excitations. To assess the plasmonic effects of the metallic nanograting, the confinement of light in the subwavelength region can be determined according to the color bar representing each point's electric field strength in the density plot. The summation of electric field components shown in Figure 10 confirms the better power (energy) concentration in the small subwavelength area of trapezoidal grooves in comparison with 
the rectangular nanogratings and the conventional type device without the nanogratings.

\section{Conclusion}

In conclusion, plasmonic-based, grating-assisted MSM-PD is supposed to be more efficient in light absorption compared with a conventional MSM-PD. Based on the SPP interaction and Fabry-Perot resonances, the power concentration in the device active layer was calculated. We have undertaken a detailed investigation of the effects of different nanograting shapes and other geometrical parameters. The analyses were based on the FDTD simulation results, and it shows $\sim 32.7-$ times optical absorption in comparison with the conventional MSM-PDs. Also, the concept of plasmonic lenses has been introduced, which provides very high optical properties for future applications. From the density plot, the near field concentration for the case of trapezoidal and rectangular nanograting structures with optimized parameters are proven to be more efficient compared with the basic MSM-PDs. These simulation results are useful for the design and development of high responsivity-bandwidth-product MSM photodetectors.

\section{References}

[1] S. Collin, F. Pardo, R. Teissier, and J. Pelouard, "Horizontal and vertical surface resonances in transmission metallic gratings," Journal of Optics A, vol. 4, pp. 154-160, 2002.

[2] E. Ozbay, "Plasmonics: merging photonics and electronics at nanoscale dimensions," Science, vol. 311, no. 5758, pp. 189-193, 2006.

[3] N. Das, A. Karar, M. Vasiliev, C. L. Tan, K. Alameh, and Y. T. Lee, "Analysis of nano-grating-assisted light absorption enhancement in metal-semiconductor-metal photodetectors patterned using focused ion-beam lithography," Optics Communications, vol. 284, no. 6, pp. 1694-1700, 2011.

[4] A. D. Zebentout, Z. Bensaad, M. Zegaoui, A. Aissat, and D. Decoster, "Effect of dimensional parameters on the current of MSM photodetector," Microelectronics Journal, vol. 42, no. 8, pp. 1006-1009, 2011.

[5] C. Cha, Broad-band and scalable circuit-level model of MSM PD for co-design with preamplifier in front-end receiver applications [Ph.D. thesis], Department of Electrical and Computer Engineering, Georgia Institute of Technology, Atlanta, Ga, USA, 2004.

[6] T. Wijesinghe and M. Premaratne, "Dispersion relation for surface plasmon polaritons on a Schottky junction," Optics Express, vol. 20, no. 7, pp. 7151-7164, 2012.

[7] L. Martín-Moreno, F. J. Garcia-Vidal, H. J. Lezec, A. Degiron, and T. W. Ebbesen, "Theory of highly directional emission from a single subwavelength aperture surrounded by surface corrugations," Physical Review Letters, vol. 90, no. 16, 4 pages, 2003.

[8] N. Das, A. Karar, M. Vasiliev, C. L. Tan, K. Alameh, and Y. T. Lee, "Groove shape-dependent absorption enhancement of $850 \mathrm{~nm}$ MSM photodetectors with nano-gratings," in Proceedings of the 10th IEEE Conference on Nanotechnology (NANO '10), pp. 289293, Seoul, Republic of Korea, August 2010.

[9] F. F. Masouleh, N. Das, and H. Mashayekhi, "Impact of duty cycle and nano-grating height on the light absorption of plasmonics-based MSM photodetectors," in Proceedings of the 12th
IEEE International Conference on Numerical Simulation of Optoelectronic Devices, pp. 13-14, Shanghai, China, 2012.

[10] T. W. Ebbesen, H. J. Lezec, H. F. Ghaemi, T. Thio, and P. A. Wolff, "Extraordinary optical transmission through sub-wavelenght hole arrays," Nature, vol. 391, no. 6668, pp. 667-669, 1998.

[11] H. J. Lezec, A. Degiron, E. Devaux et al., "Beaming light from a subwavelength aperture," Science, vol. 297, no. 5582, pp. 820822, 2002.

[12] Y. Ding, J. Yoon, M. H. Javed, S. H. Song, and R. Magnusson, "Mapping surface-plasmon polaritons and cavity modes in extraordinary optical transmission," IEEE Photonics Journal, vol. 3, no. 3, pp. 365-374, 2011.

[13] S. A. Maier, Plasmonics: Fundamentals and Applications, Springer, New York, NY, USA, 2007.

[14] G. T. Reed and A. P. Knight, Silicon Photonics: An Introduction, John Wiley and Sons, New York, NY, USA, 2004.

[15] D. Dhakal, Analysis of the sub-wavelength grating in OptiFDTD simulator [M.S. thesis], School of Engineering and Science, Jacobs University, Bremen, Germany, 2009.

[16] H. Raether, Surface Plasmons on Smooth and Rough Surfaces and on Gratings, Springer, Berlin, Germany, 1988.

[17] D. Mawet, P. Riaud, J. Baudrand et al., "Achromatic optical vortex coronagraph with subwavelength gratings," in Proceedings of the International Coronagraph Workshop, pp. 69-72, Pasadena, Calif, USA, 2006.

[18] H. Kikuta, H. Toyota, and W. Yu, "Optical elements with subwavelength structured surfaces," Optical Review, vol. 10, no. 2, pp. 63-73, 2003.

[19] J. S. White, G. Veronis, Z. Yu et al., "Extraordinary optical absorption through subwavelength slits," Optics Letters, vol. 34, no. 5, pp. 686-688, 2009.

[20] D. de Ceglia, M. A. Vincenti, M. Scalora, N. Akozbek, and M. J. Bloemer, "Enhancement and inhibition of transmission from metal gratings: engineering the spectral response," 2010, http:// arxiv.org/abs/1006.3841.

[21] J. A. Porto, F. J. García-Vidal, and J. B. Pendry, "Transmission resonances on metallic gratings with very narrow slits," Physical Review Letters, vol. 83, no. 14, pp. 2845-2848, 1999.

[22] T. López-Rios, D. Mendoza, F. J. García-Vidal, J. SánchezDehesa, and B. Pannetier, "Surface shape resonances in lamellar metallic gratings," Physical Review Letters, vol. 81, no. 3, pp. 665$668,1998$.

[23] A. Barbara, P. Quémerais, E. Bustarret, and T. Lopez-Rios, "Optical transmission through subwavelength metallic gratings," Physical Review B, vol. 66, no. 16, Article ID 161403, 4 pages, 2002.

[24] R. Gordon, D. Sinton, K. L. Kavanagh, and A. G. Brolo, "A new generation of sensors based on extraordinary optical transmission," Accounts of Chemical Research, vol. 41, no. 8, pp. 10491057, 2008.

[25] Y. Xie, A. R. Zakharian, J. V. Moloney, and M. Mansuripur, "Transmission of light through a periodic array of slits in a thick metallic film," Optics Express, vol. 13, no. 12, pp. 4485-4491, 2005.

[26] K. S. Yee, "Numerical solution of initial boundary value problems involving maxwell's equations in isotropic media," IEEE Transactions on Antennas and Propagation, vol. 14, p. 302, 1966.

[27] A. Bondeson, T. Rylander, and P. Ingelstrom, Computational Electromagnetics, Springer, New York, NY, USA, 1st edition, 2005. 
[28] R. Marani, M. Grande, V. Petruzzelli, and A. D’Orazio, "Plasmonic bandgaps in 1D arrays of slits on metal layers excited by out-of-plane sources," International Journal of Optics, vol. 2012, Article ID 146396, 12 pages, 2012.

[29] M. Bera and M. Ray, "Role of waveguide resonance in coupled plasmonic structures using bimetallic nanofilms," Optical Engineering, vol. 51, no. 10, Article ID 103801, 2012.

[30] N. Zhang, R. Schweiss, Y. Zong, and W. Knoll, "Electrochemical surface plasmon spectroscopy-recent developments and applications," Electrochimica Acta, vol. 52, no. 8, pp. 2869-2887, 2007.

[31] C. Genet and T. W. Ebbesen, "Light in tiny holes," Nature, vol. 445, no. 7123, pp. 39-46, 2007.

[32] A. S. Vengurlekar, "Extraordinary optical transmission through metal films with subwavelength holes and slits," Current Science, vol. 98, no. 8, pp. 1020-1032, 2010.

[33] B. Sturman, E. Podivilov, and M. Gorkunov, "Theory of extraordinary light transmission through arrays of subwavelength slits," Physical Review B, vol. 77, no. 7, Article ID 075106, 2008.

[34] R. Umeda, C. Totsuji, K. Tsuruta, and H. Totsuji, "An FDTD analysis of nanostructured electromagnetic metamaterials using parallel computer," Materials Transactions, vol. 50, no. 5, pp. 994-998, 2009.

[35] A. D. Rakić, A. B. Djurišić, J. M. Elazar, and M. L. Majewski, "Optical properties of metallic films for vertical-cavity optoelectronic devices," Applied Optics, vol. 37, no. 22, pp. 5271-5283, 1998.

[36] M. Bordovsky, P. Catrysse, S. Dods et al., "Waveguide design, modeling, and optimization: from photonic nanodevices to integrated photonic circuits," in Integrated Optics: Devices, Materials, and Technologies VIII, 65, vol. 5355 of Proceedings of SPIE, San Jose, Calif, USA, May 2004.

[37] B. Ung, Study of the interaction of surface waves with a metallic nano-slit via the finite-difference time-domain method [M.S. thesis], Laval University, Quebec, Canada, 2007.

[38] J. A. Schuller, E. S. Barnard, W. Cai, Y. C. Jun, J. S. White, and M. L. Brongersma, "Plasmonics for extreme light concentration and manipulation," Nature Materials, vol. 9, pp. 193-204, 2010.

[39] Y. Fu and X. Zhou, "Plasmonic lenses: a review," Plasmonics, vol. 5, no. 3, pp. 287-310, 2010.

[40] R. Grote, R. M. Osgood, J. E. Spanier, and B. Nabet, "Optimization of a surface plasmon enhanced metal-semiconductormetal photodetector on Gallium Arsenide," in Proceedings of the Frontiers in Optics, OSA Technical Digest (CD) (Optical Society of America), Paper FThY3, Rochester, NY, USA, October 2010.

[41] N. Das, A. Karar, C. L. Tan, K. Alameh, and Y. T. Lee, "Impact of nanograting phase-shift on light absorption enhancement in plasmonics-based metal-semiconductor-metal photodetectors," Advances in Optical Technologies, vol. 2011, Article ID 504530, 8 pages, 2011. 

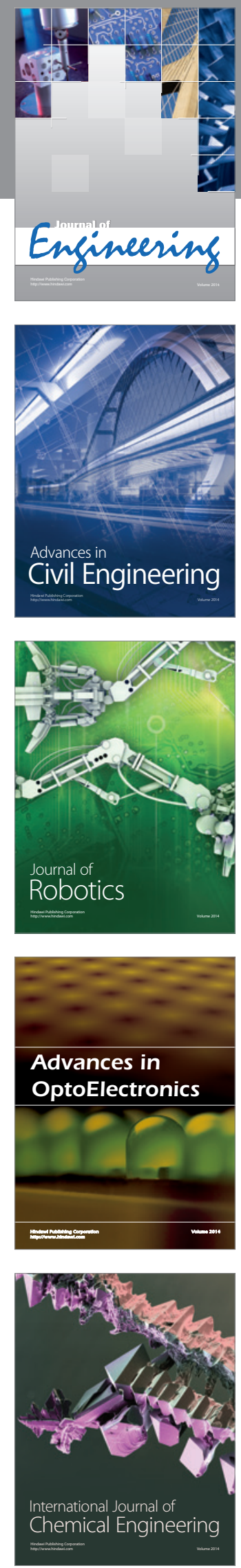

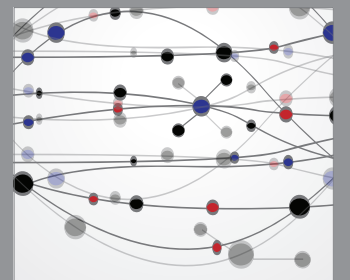

The Scientific World Journal
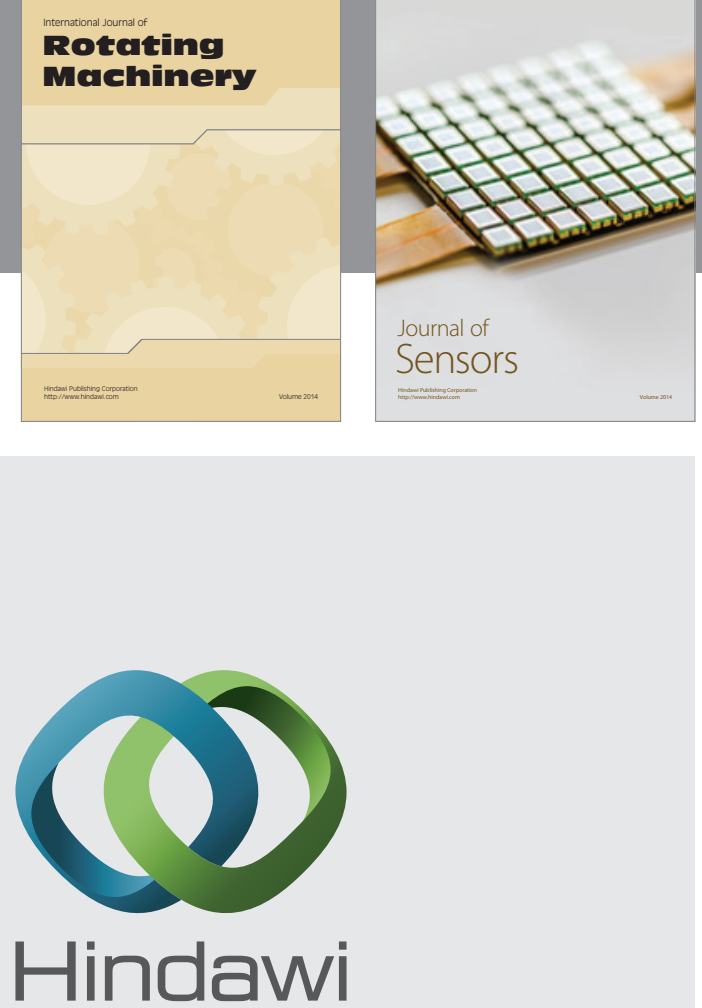

Submit your manuscripts at http://www.hindawi.com
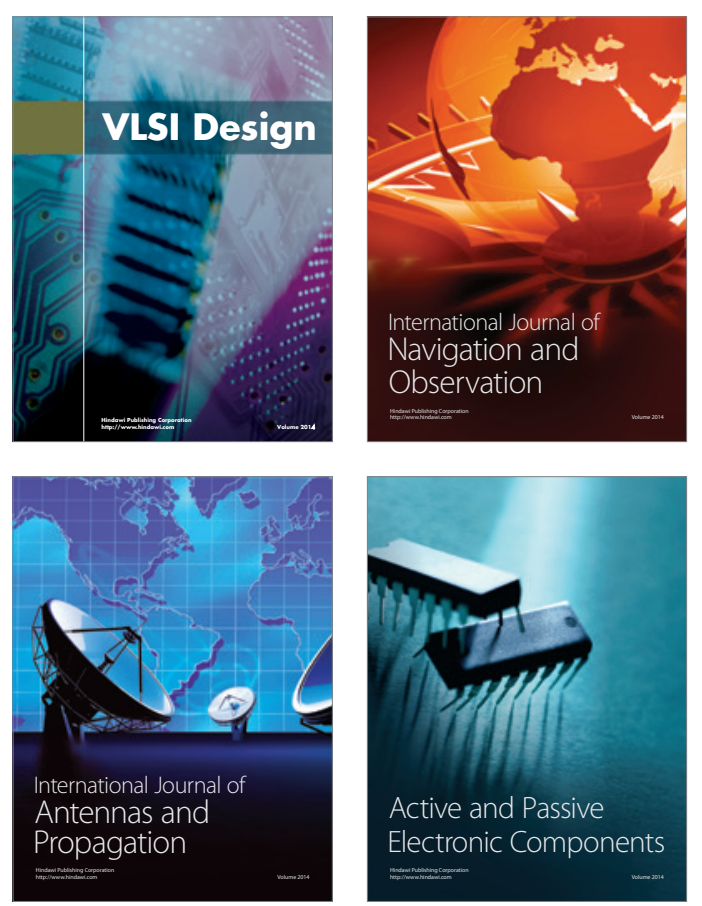
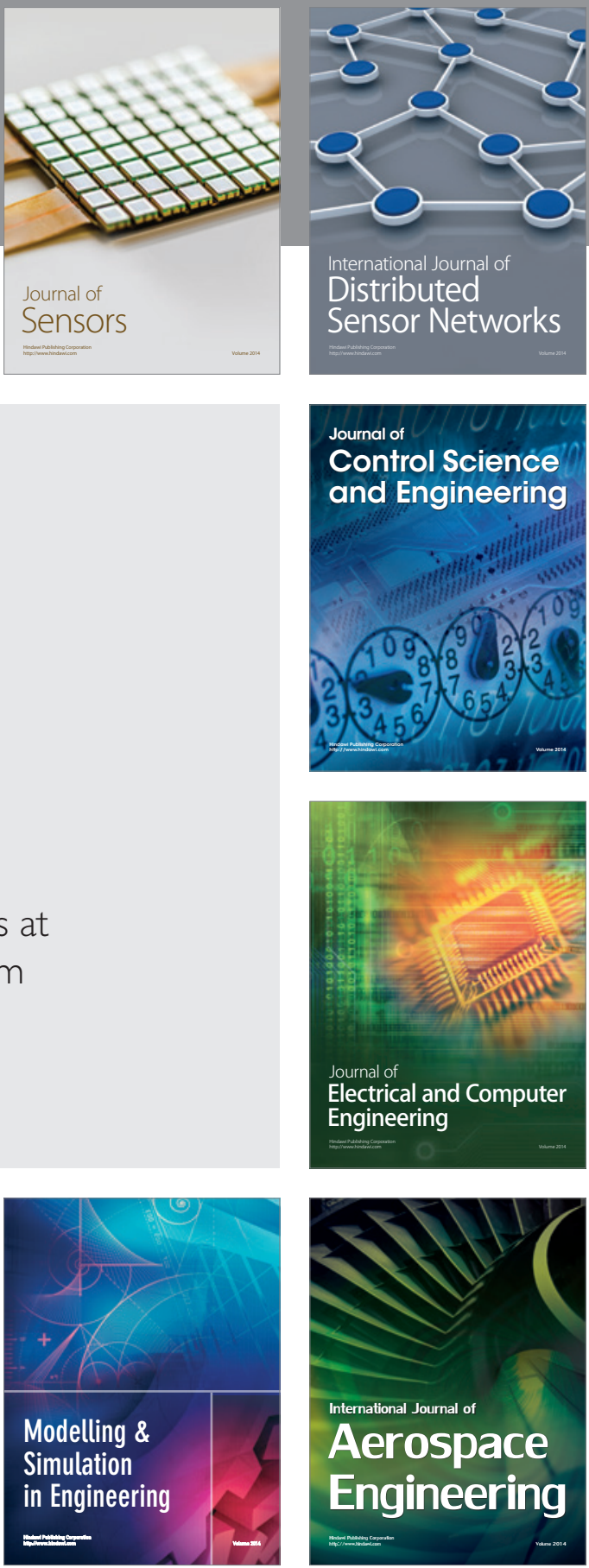

Journal of

Control Science

and Engineering
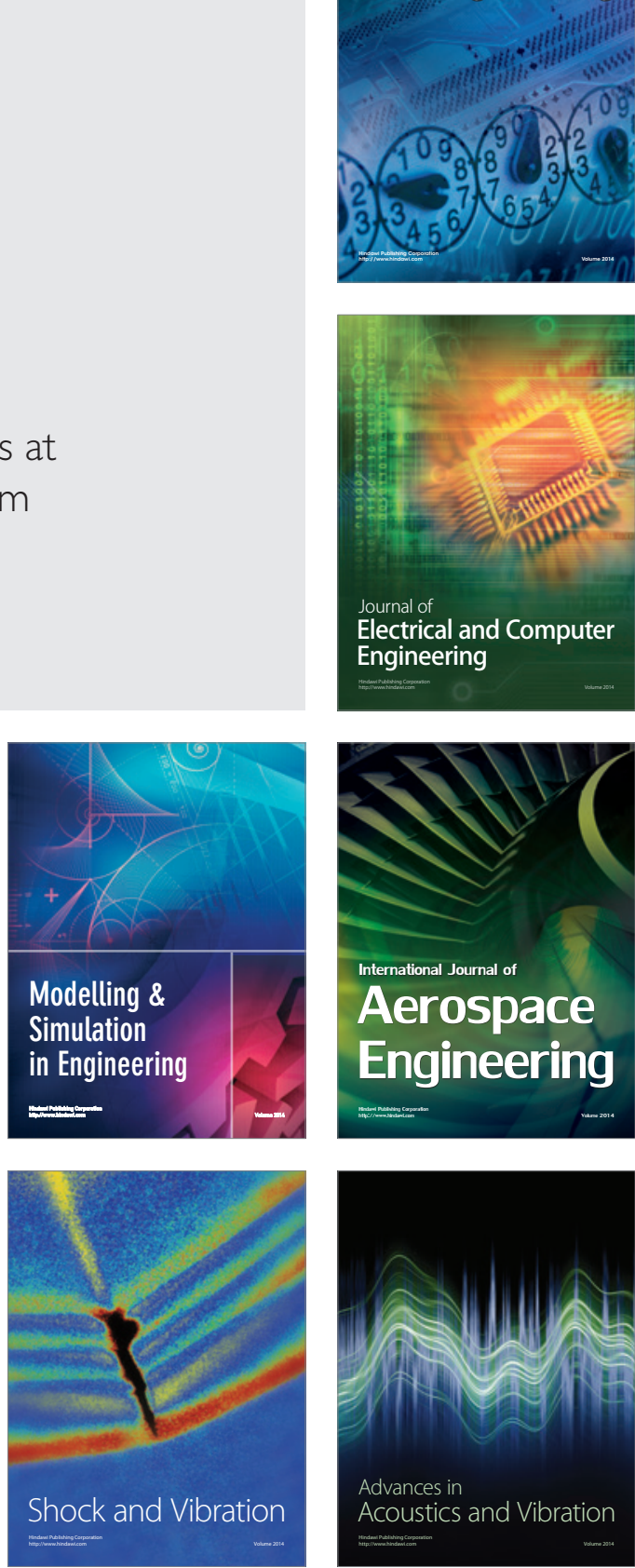